\title{
Inventario preliminar y uso de mamíferos silvestres no voladores en la vereda Camarón, Montes de María (Bolívar-Colombia)
}

\author{
Angie N. Tinoco-Sotomayor \\ atinocos@unicartagena.edu.co

\section{Hayder D. Ramos-Guerra \\ Hugo A. Vides-Avilez} \\ D. Carolina Rodríguez-Alarcón
}

Grupo de Investigación en Química de Medicamentos, línea de investigación en ecología y conservación de flora y fauna silvestre. Programa de Biología, Facultad de Ciencias Exactas y Naturales, Universidad de Cartagena, Cartagena-Colombia.

Grupo de Investigación en Química de Medicamentos, línea de investigación en ecología y conservación de flora y fauna silvestre. Programa de Biología, Facultad de Ciencias Exactas y Naturales, Universidad de Cartagena, Cartagena-Colombia.

José F. González-Maya

Proyecto de Conservación de Aguas y Tierras, ProCAT Colombia, Carrera 13 \# 96 - 82, Of. 205, Bogotá, Colombia \& Instituto de Ecología, Universidad Nacional Autónoma de México, México.

\section{Harold Gómez-Estrada}

Grupo de Investigación en Química de Medicamentos, línea de investigación en ecología y conservación de flora y fauna silvestre. Programa de Química Farmacéutica, Facultad de Ciencias Farmacéuticas, Universidad de Cartagena, Cartagena-

Colombia.

Colombia es el sexto país en diversidad de mamíferos en el mundo, con 518 especies nativas registradas a la fecha (RamírezChaves et al. 2016). Para el bosque seco tropical (BST) del Caribe colombiano se han registrado un total de nueve órdenes, 26 familias y 60 especies de mamíferos, lo que corresponde al 12\% de la diversidad nacional (Pizano \& García 2014). La riqueza de especies y la diversidad de mamíferos en el bosque seco se consideran menor que en los bosques húmedos. Sin embargo, el conocimiento de los mamíferos del BST en el país es escaso y los estudios se han realizado en pequeñas extensiones geográficas o zonas muy intervenidas, y en general de corto tiempo (Pizano \& García 2014).

Dentro de las principales amenazas sobre los mamíferos, la cacería es potencialmente una de las que ha contribuido de forma fundamental a la reducción de sus poblaciones a nivel global (Ceballos \& Ehrlich 2002, Schipper et al. 2008). Sin embargo, esta es una de las actividades más relacionadas con las poblaciones humanas rurales, siendo múltiples las razones para su práctica (e.g., nutrición, fuente de ingresos, valor medicinal o espiritual; Altrichter 2006, Chacón P. \& González-Maya 2013). El efecto de la cacería tiene implicaciones en las poblaciones de muchos mamíferos, siendo uno de los principales conductores de extinciones locales o de reducción de las poblaciones (Ceballos \& Ehrlich 2002, Ceballos et al. 2010), lo que trae consecuencias a largo plazo sobre el funcionamiento de los ecosistemas y los modos de vida de la gente.

En Colombia la cacería es una de las principales amenazas sobre la biodiversidad, en especial de los mamíferos (BalagueraReina \& González-Maya 2007, González-Maya et al. 2010, González-Maya et al. 2011, Arias Alzate et al. 2012, Chacón P. \& González-Maya 2013, González-Maya et al. 2013a, González-Maya et al. 2013b, González-Maya et al. 2013d), por lo que es necesario entender sus dimensiones para una adecuada planificación de conservación. A la fecha, pocos estudios se han enfocado en caracterizar esta amenaza a nivel nacional. El objetivo de este trabajo fue identificar de forma preliminar los mamíferos no voladores presentes en la vereda Camarón, departamento de Bolívar, Colombia y su uso por parte de las comunidades locales.

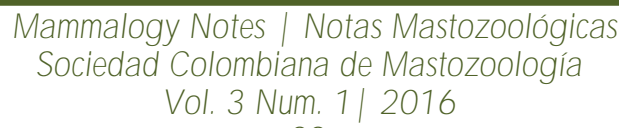


El área de estudio se encuentra ubicada en la vereda Camarón (0950'32.5" N 075¹7'37.4" W), municipio de El Cármen de Bolívar, subregión Montes de María, en el departamento de Bolívar (Figura 1); la vereda posee elevaciones máximas de 1200 $\mathrm{msnm}$, temperaturas que oscilan entre 26 y $30^{\circ} \mathrm{C}$, y una precipitación anual promedio de $1500 \mathrm{~mm}$ (ProMontes 2003). Se caracteriza por presentar principalmente parches de bosque seco tropical en estado de sucesión, vegetación arbustiva, bosques de galería, cultivos agrícolas permanentes y estacionales, e incursiones de cultivos de palma de aceite y zonas deforestadas para actividad pastoril (Pizano \& García 2014).

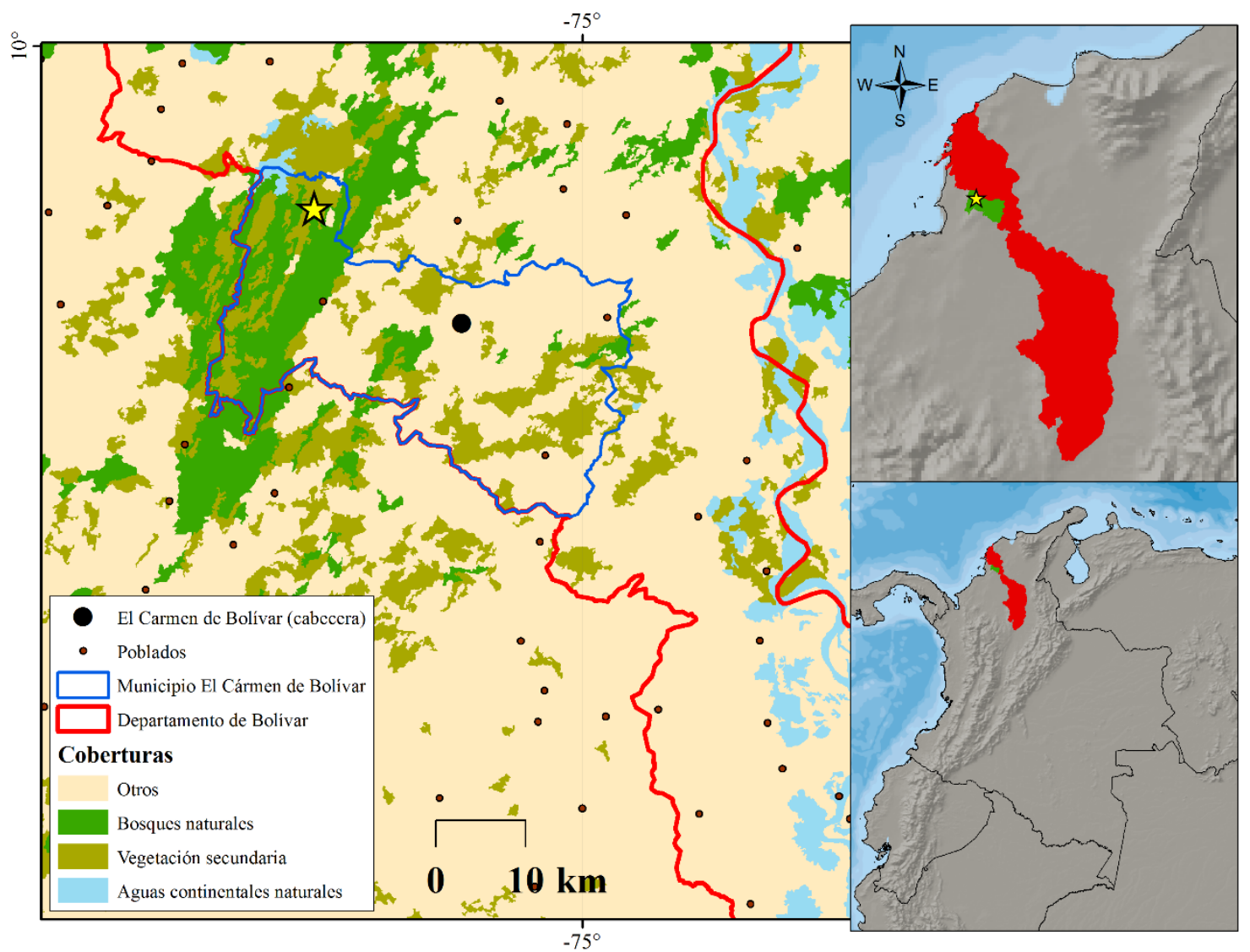

Figura 1. Localización de la vereda Camarón, subregión Montes de María, departamento de Bolívar, Colombia, donde se evaluó la riqueza y uso de mamíferos.

Durante los meses de julio y agosto de 2015 y marzo de 2016, se realizaron visitas de campo a la vereda Camarón, como parte del proyecto "Hacia el Conocimiento y Estudio de la Fauna Silvestre en la Vereda Camarón y el Municipio Santo Domingo de Meza, Montes de María (Bolívar)." Se realizaron rastreos directos en 5 transectos lineales de $1 \mathrm{~km}$, dos repeticiones en cada uno, con el fin de obtener una lista preliminar de especies de mamíferos de la zona. Asimismo, se realizaron 140 entrevistas semiestructuradas a la comunidad para determinar los usos que las comunidades dan a estas especies. Las entrevistas se realizaron preferiblemente a cazadores y se utilizaron guías de campo de mamíferos (Emmons \& Feer 1999, Aranda 2012) así como fichas con dibujos y fotografías de los mamíferos presentes en el Caribe colombiano (RaceroCasarrubia \& González-Maya 2014).

Se registraron 27 especies de mamíferos distribuidos en ocho órdenes y 20 familias, de los cuales el $29 \%$ son cazados para consumo, el $37 \%$ son cazados para control, el $25.9 \%$ es usado como mascota y el $14.8 \%$ no presentan ningún uso (Tabla 1;

\section{Mammalogy Notes | Notas Mastozoológicas \\ Sociedad Colombiana de Mastozoología \\ Vol. 3 Num. 1| 2016}


Figura 2). Especies como el Ñeque (Dasyprocta punctata) y la Guartinaja (Cuniculus paca) son las más consumidas por la comunidad, considerados los más fáciles de cazar, seguidos por el Zaino (Pecari tajacu) y el Conejo (Sylvilagus floridanus).

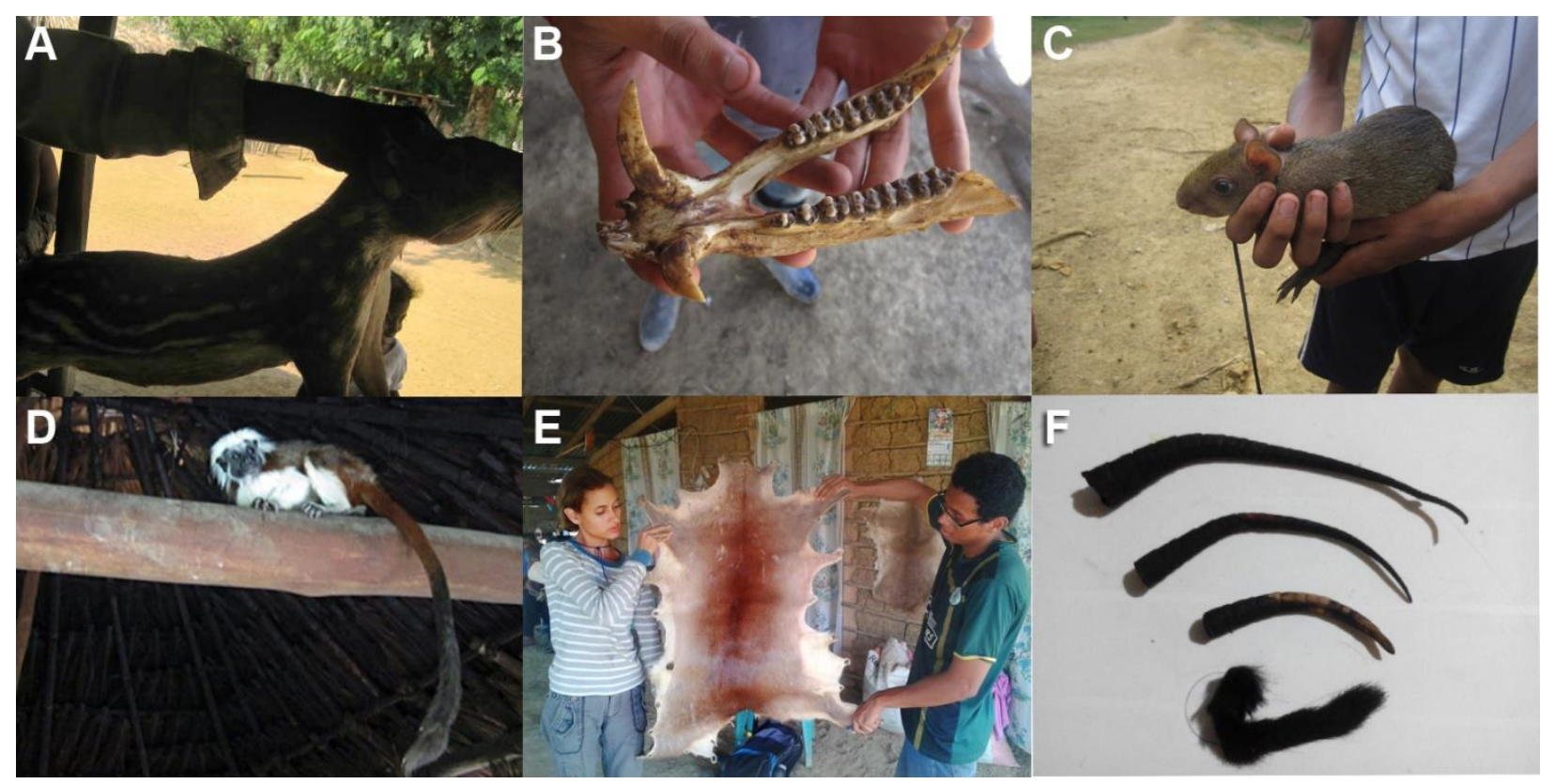

Figura 2. Registro de especies de mamíferos medianos y grandes a partir de restos obtenidos de las comunidades de en la vereda Camarón, Montes de María, Bolívar, Colombia. A. Guartinaja (Cuniculus paca); B. Zaino (Pecari tajacu); C. Ñeque (Dasyprocta punctata); D. Tití cabeciblanco (Saguinus oedipus); E. Venado (Mazama sp.); F. Ardilla (Sciurus granatensis) y Armadillo de Nueve Bandas (Dasypus novemcinctus),

Entre los más apetecidos por la comunidad se citan los Venados (Mazama sp. y Odocoileus virginianus), siendo difíciles de obtener en la zona. Una de las especies que se consideran menos frecuentes pero apetecidas es el Armadillo trueno o Cola de trapo Cabassous centralis, que para obtenerlo se requiere recorrer grandes distancias, específicamente a las zonas más montañosas, como ya fue reportado para la región (Cruz-Rodríguez et al. 2011). El consumo de subsistencia predomina sobre el comercio de carne de monte, donde sólo una parte es vendida. Se encontró que aún existe un mercado de carne silvestre, principalmente dirigido a los municipios de Carmen de Bolívar, San Jacinto, San Juan Nepomuceno y María la Baja. Sin embargo, sólo el 10\% de los entrevistados indicó que la cacería es su principal actividad económica.

Las especies de mamíferos que presentan algún conflicto con la comunidad, y las cuales son controladas, son principalmente del orden Carnivora, en donde el conflicto ha sido principalmente por el consumo de aves de corral, resaltando el manejo libre que se les da en la zona. Las especies cazadas más frecuentemente por esta causa incluyen Eira barbara, Cerdocyon thous, seguidos por Leopardus pardalis y L. wiedii. Existen conflictos de menor escala por encuentros con perros (i.e., Tamandua mexicana) y daños a cultivos (i.e., Sciurus granatensis y Procyon cancrivorus), así como especies carismáticas tenidas como mascotas (e.g., Bradypus variegatus, Saguinus oedipus, Alouatta seniculus, Pecari tajacu y Mazama sp.). Adicionalmente, se registró presencia histórica de especies como el jaguar (Panthera onca), con un presunto avistamiento hace seis años coincidente con registros previos para la zona (Chacón et al. 2014), y un avistamiento de puma (Puma concolor) hace cinco años, y una presunta depredación de $C$. centralis en 2014, aunque se consideran especies extintas en la vereda.

Los Montes de María, en el Caribe Colombiano, posee relictos de bosques poco intervenidos, que debido a factores sociales se han mantenido, pero actualmente están siendo de nuevo presionados por expansión agrícola y altos niveles de cacería (ProMontes 2003, Cruz-Rodríguez et al. 2011). A pesar de estas amenazas, la ecorregión es aún considerada uno de los últimos remanentes prioritarios de bosque seco para el Caribe colombiano (González-Maya et al. 2013c, Larrotta et al. 2016), aunque actualmente no cuenta con ninguna figura oficial de conservación (i.e., áreas protegidas). La falta de políticas públicas

\section{Mammalogy Notes | Notas Mastozoológicas \\ Sociedad Colombiana de Mastozoología \\ Vol. 3 Num. 1| 2016}


adecuadas, la ausencia de figuras oficiales de conservación, y la poca presencia y control de autoridades ambientales, hacen que la región se encuentre amenazada actualmente, a pesar de 25 años de conflicto social que permitieron recuperar una significativa proporción de este territorio. Dado que la cacería insostenible sigue siendo una de las principales actividades en la región, a pesar de no ser la fuente primaria de ingresos para la mayoría de entrevistados, es necesario explorar las dimensiones de la actividad, con el fin de que se incluya esta información en la adecuada planificación de conservación de la ecorregión.

Tabla 1. Mamíferos registrados para la vereda Camarón y sus cerros, Montes de María, Bolívar. Tipo de registros: EN: Entrevistas; RV: Registro visual; P: Piel; RC: Registro colectado; V: Vocalización. Uso: C: Consumo; M: Mascotas; T: Control

\begin{tabular}{|c|c|c|c|c|}
\hline Orden & Familia & Nombre Común & Tipo de registro & Uso \\
\hline \multirow[t]{4}{*}{ Didelphimorphia } & Didelphidae & & & \\
\hline & Didelphis marsupialis & Zorra Chucha o zorra pela & EN & $\mathrm{T}$ \\
\hline & Didelphis albiventris & Zorra Chucha o zorra pela & RV & $\mathrm{T}$ \\
\hline & Dasypodidae & & & \\
\hline \multirow[t]{5}{*}{ Cingulata } & Dasypus novemcinctus & Armadillo fino o blanco & $\mathrm{RC}$ & $\mathrm{C}$ \\
\hline & Cabassous centralis & Armadillo cola de trapo, & EN & $\mathrm{C}$ \\
\hline & Bradypodidae & & & \\
\hline & Bradypus variegatus & Perezoso & RV & $\mathrm{M}$ \\
\hline & Choloepidae & & & \\
\hline \multirow{6}{*}{ Pilosa } & Choloepus hoffmanni & Perico ligero o perezoso mono & EN & \\
\hline & Myrmecophagidae & & & \\
\hline & Myrmecophaga tridactyla & Oso cola de caballo & EN & \\
\hline & Tamandua mexicana & Oso hormiguero & EN & $\mathrm{T}$ \\
\hline & Callitrichinae & & & \\
\hline & Saguinus oedipus & Titi & RV & M \\
\hline \multirow[t]{4}{*}{ Primates } & Atelidae & & & \\
\hline & Alouatta seniculus & Mono colorado & $\mathrm{RV}, \mathrm{V}$ & $\mathrm{M}$ \\
\hline & Aotidae & & & \\
\hline & Aotus griseimembra & Martas & RV & \\
\hline \multirow[t]{8}{*}{ Rodentia } & Sciuridae & & & \\
\hline & Sciurus granatensis & Ardita & $\mathrm{RV}, \mathrm{RC}$ & $\mathrm{T}$ \\
\hline & Herethizontidae & & & \\
\hline & Coendou sanctamartae & Puercoespín & EN & $\mathrm{C}$ \\
\hline & Dasyproctidae & & & \\
\hline & Dasyprocta punctata & Neque & RV & $\mathrm{C}, \mathrm{M}$ \\
\hline & Cuniculidae & & & \\
\hline & Cuniculus paca & Guartinaja, Guagua & RV & $\mathrm{C}, \mathrm{M}$ \\
\hline \multirow[t]{3}{*}{ Lagomorpha } & Leporidae & & & \\
\hline & Sylvilagus floridanus & Conejo & RV & $\mathrm{C}$ \\
\hline & Felidae & & & \\
\hline \multirow[t]{10}{*}{ Carnivora } & Leopardus pardalis & Tigrillo & EN & $\mathrm{T}$ \\
\hline & Leopardus wiedii & Gato pardo o de monte & EN & $\mathrm{T}$ \\
\hline & Canidae & & & \\
\hline & Cerdocyon thous & Zorra baya & EN & $\mathrm{T}$ \\
\hline & Speothos venaticus & Perrito de monte & EN & $\mathrm{T}$ \\
\hline & Mustelidae & & & \\
\hline & $\begin{array}{l}\text { Eira barbara } \\
\text { Procyonidae }\end{array}$ & Guacharo, cocoromao & EN & $\mathrm{T}$ \\
\hline & Procyon cancrivorus & Pata de muchacho & EN & $\mathrm{T}$ \\
\hline & Mephitidae & & & \\
\hline & Conepatus semistriatus & Mapurito, zorrillo & EN & \\
\hline \multirow[t]{5}{*}{ Artiodactyla } & Tayassuidae & & & \\
\hline & Pecari tajacu & Zaino & RV & $\mathrm{C}, \mathrm{M}$ \\
\hline & Cervidae & & & \\
\hline & Mazama americana & Venado & $\mathrm{P}$ & $\mathrm{C}, \mathrm{M}$ \\
\hline & Odocoileus virginianus & Venado & EN & $\mathrm{C}$ \\
\hline
\end{tabular}

\footnotetext{
Mammalogy Notes | Notas Mastozoológicas

Sociedad Colombiana de Mastozoología

Vol. 3 Num. 1| 2016
} 


\title{
Inventarios | Inventories
}

Agradecimientos

Agradecemos a la comuni dad dela vereda Camarón, miembros del proyecto “Hacia el Conocimiento y Estudio dela Fauna Silvestre en la Vereda Camarón y el Municipio Santo Domingo de Meza, Montes de María (Bolívar)”, y a M. E. Guardo, J. M. Sánchez y E. Torres Niño, por su apoyo.

\section{Referencias}

ALTRICHTER, M. 2006. Wildlife in the life of local people of the semi-arid Argentine Chaco. Biodiversity and Conservation 15: 2719-2736.

ARANDA, M. 2012. Manual para el rastreo de mamíferos silvestres de México. Comisión Nacional para el conocimiento y uso de la biodiversidad (Conabio), México DF, Mexico 258.

ARIAS ALZATE, A., et al. 2012. Conservación de mamíferos del Chocó: distribución, riqueza y representatividad de las áreas protegidas. Revista Biodiversidad Neotropical 2: 69-80.

BALAGUERA-REINA, S. A. \& J. F. GONZÁLEZ-MAYA. 2007. Occasional Jaguar Hunting for Subsistence in Colombian Chocó. CatNews 48: $122-122$.

CEBALLOS, G. \& P. R. EHRLICH. 2002. Mammal population losses and the extinction crisis. Science 296: 904-907.

CEBALLOS, G., et al. 2010. The sixth extinction crisis loss of animal populations and species. Science 8: 1821-1831.

CRUZ-RODRÍGUEZ, C. A., et al. 2011. New records for the Northern Naked-Tailed Armadillo Cabassous centralis (Cingulata: Dasypodidae) in Tropical Dry Forests of the Department of Sucre, Colombian Caribbean. Revista Mexicana de Mastozoología 15: 39-45.

CHACÓN P. J. \& J. F. GONZÁLEZ-MAYA. 2013. Noteworthy record of subsistence hunting and meat consumption of Jaguarundi (Puma yagouarundi) in Colombia. Revista Mexicana de Mastozoología 3: 10-13.

CHACÓN P. J., et al. 2014. Reaparición del jaguar (Panthera onca) en el departamento de Sucre, Colombia. Mammalogy Notes 1: 8-9.

EMMONS, L. H. \& F. FEER. 1999. Neotropical Rainforest Mammals: A Field Guide, Second Edition. The University of Chicago Press, Chicago, IL, United States 320-320. GONZÁLEZ-MAYA, J. F., et al. 2011. Research priorities for the small carnivores of Colombia. Small Carnivore Conservation 44: 7-13.

GONZÁLEZ-MAYA, J. F., et al. 2013a. Conflictos felinos-vida silvestre en el Caribe Colombiano: un estudio de caso en los departamentos del Cesar y La Guajira. Pp. 51-59 en Plan de Conservación de Felinos del Caribe Colombiano: Los felinos y su papel en la planificación regional integral basada en especies clave (Castaño-Uribe C, González-Maya JF, Zárrate-Charry DA, Ange-Jaramillo C, and Vela-Vargas IM eds.), Fundación Herencia Ambiental Caribe, ProCAT Colombia, The Sierra to Sea Institute, Santa Marta, Colombia.

GONZÁLEZ-MAYA, J. F., et al. 2013b. Records of occasional puma hunting for consumption in Colombia. CatNews 58: 9-10.

GONZÁLEZ-MAYA, J. F., et al. 2013c. Evaluación geográfica y prioridades de conservación de hábitat para felinos en el Caribe colombiano. Pp. 77-87 en Plan de Conservación de Felinos del Caribe Colombiano: Los felinos y su papel en la planificación regional integral basada en especies clave (Castaño-Uribe C, González-Maya JF, Zárrate-Charry DA, Ange-Jaramillo C, and Vela-Vargas IM eds.), Fundación Herencia Ambiental Caribe, ProCAT Colombia, The Sierra to Sea Institute, Santa Marta, Colombia.

GONZÁLEZ-MAYA, J. F., et al. 2013d. Ecología y conservación de felinos y presas en el Caribe colombiano. Pp. 95-104 en Plan de Conservación de Felinos del Caribe Colombiano: Los felinos y su papel en la planificación regional integral basada en especies clave (Castaño-Uribe C, González-Maya JF, Zárrate-Charry DA, Ange-Jaramillo C, and Vela-Vargas IM eds.), Fundación Herencia Ambiental Caribe, ProCAT Colombia, The Sierra to Sea Institute, Santa Marta, Colombia.

GONZÁLEZ-MAYA, J. F., et al. 2010. Traditional uses of wild felids in the Caribbean region of Colombia: new threats for conservation? Revista Latinoamericana de Conservación 1: 64-69.

LARROTTA, L., et al. 2016. Primates en un paisaje fragmentado de los Montes de María, Colombia. Editorial Académica Española, Saarbrücken, Germany.

PIZANO, C. \& H. GARCÍA. 2014. El Bosque Seco Tropical en Colombia. Instituto de Investigación de Recursos Biológicos Alexander von Humboldt (IAvH), Bogotá D.C., Colombia 352

PROMONTES. 2003. Programa de Desarrollo y Paz de los Montes de María - Promontes. PNUD, Corporación Territorios, Universidad de Cartagena.

RACERO-CASARRUBIA, J. \& J. F. GONZÁLEZ-MAYA. 2014. Inventario preliminar y uso de mamíferos silvestres por comunidades campesinas del sector oriental del cerro Murrucucú, municipio de Tierralta, Córdoba, Colombia. Mammalogy Notes 1: 25-28.

RAMÍREZ-CHAVES, H. E. et al. 2016. Cambios recientes a la lista de los mamíferos de Colombia. Mammalogy Notes 3(1): 1-9.

SCHIPPER, J., et al. 2008. The status of the world's land and marine mammals: diversity, threat, and knowledge. Science 322: 225-230.

\author{
Mammalogy Notes | Notas Mastozoológicas \\ Sociedad Colombiana de Mastozoología \\ Vol. 3 N um. 1| 2016
}

